\title{
MEASUREMENT TECHNIQUES FOR THE OPTICAL QUALITY ASSESSMENT OF PARABOLIC TROUGH COLLECTOR FIELDS IN COMMERCIAL SOLAR POWER PLANTS
}

\author{
Steffen UImer \\ German Aerospace Center (DLR) \\ Institute of Technical Thermodynamics \\ Solar Research, Plataforma Solar de Almería \\ 04200 Tabernas, Spain
}

\author{
Eckhard Lüpfert \\ German Aerospace Center (DLR) \\ Institute of Technical Thermodynamics \\ Solar Research, 51170 Cologne, Germany
}

\author{
Klaus Pottler \\ German Aerospace Center (DLR) \\ Institute of Technical Thermodynamics \\ Solar Research, Plataforma Solar de Almería \\ 04200 Tabernas, Spain \\ Marc Röger \\ German Aerospace Center (DLR) \\ Institute of Technical Thermodynamics \\ Solar Research, Plataforma Solar de Almería \\ 04200 Tabernas, Spain
}

\begin{abstract}
The optical quality of the collector field of concentrating solar power plants is an essential factor for their profitability. High optical quality can be achieved and guaranteed when the manufacturing process is continuously monitored and adjusted in its essential steps.

A stationary, automatic photogrammetry system has been developed for the shape accuracy control of concentrator structures. It uses a digital camera, automatically moved around the object, computer controlled automatic image evaluation and continuous calibration checks. It is robust and fast enough to be integrated in a solar collector production line.

For on-site measurements of mirror slope in parabolic trough collector fields, a new method is presented. It uses a set of pictures of the reflections of the absorber tube in the concentrator. The slope errors of the mirror surface are calculated with high spatial resolution and accuracy. The effects of the reflector slope deviations on the optical performance are evaluated with ray-tracing. The results give detailed information about the optical quality of the concentrator, inaccuracies in the manufacturing process, and their optical performance penalty.
\end{abstract}

\section{INTRODUCTION}

The optical performance of solar concentrating collectors is sensitive to the geometric inaccuracies of structural and optical components and their assembly. Because of the finite sun-shape and imprecisions of the collector system (e.g. tracking, receiver alignment, mirror alignment, mirror shape and mirror specularity), the interception of the reflected sunrays at the focal receiver is below $100 \%$ (1). For the 3-dimensional measurement of points in space the application of tachymeters is possible. These systems are commonly used for surveying purposes and therefore well known and cost-effective. Drawbacks are the necessity of spherical prisms with diameters in the range of a few centimeters whose size complicates accurate measurements of collector details and an inherent distance measurement uncertainty of about $1 \mathrm{~mm}$. Close-range photogrammetry in contrast has proven to be well suited for this measurement task. The used retro-reflective photogrammetry targets have heights of only $2 \mathrm{~mm}$ and are easier and more precise to apply for probing than spherical prisms. Previous work $(2,3)$ describes the application of photogrammetry to the characterization of solar collectors. In the last years, DLR has done various photogrammetric analyses of different kinds of parabolic trough collectors $(4,5)$.

High resolution photogrammetry has also been used for the assessment of mirror shape. However, the mirror surface has to 
be prepared with a pattern of measurement points to make the measurement possible. This is a time-consuming task and the uncertainty of local slope measurement values increases with smaller point-to-point distances. In the past laser scanners were developed to measure the slope errors of parabolic troughs with high precision $(6,7)$. As these systems only give information on a line section across the aperture, several parallel scans for the assessment of large areas are necessary. This is very time consuming. This paper describes a method to directly measure surface slopes which is suitable for measuring large surfaces in an existing solar field with high resolution and low time-effort. So-called deflectometric measurement systems with a known geometric set-up, a screen with a regular pattern and a digital camera can be used to detect dents and other defects in the specular surfaces by analyzing the distortions in the reflection of the regular pattern (8). Based on this principle a measurement method for the special case of parabolic trough collectors was developed and applied.

\section{AUTOMATIC SUPPORT STRUCTURE CHECK}

The necessity of quality control in the assembly line is demonstrated by the following example. Figure 1 shows a parabolic trough structure, measured at the Plataforma Solar de Almería (PSA) that reveals systematic deviations for the second row of mirror attachment clamps (they are too low) and exhibit one point that is mounted higher than designed.

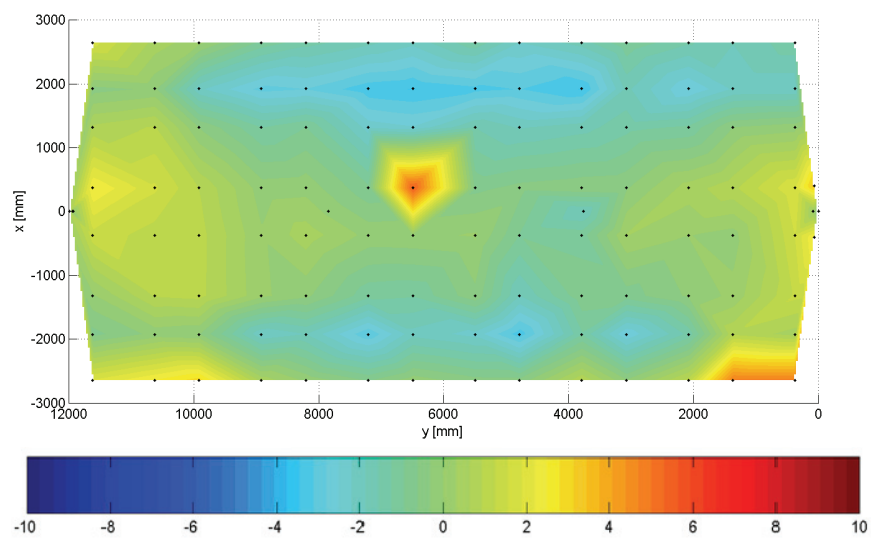

Figure 1: Photogrammetric measurement result of a parabolic trough steel structure. The height deviations from the design values of the mirror facet attachment clamps are displayed in $\mathbf{m m}$.

For the quality control of a collector assembly line, an automatic measurement system accurate enough to find assembly errors and fast enough to not disturb the regular assembling procedure is needed. This was achieved with the stationary, automatic photogrammetry system "Q-Foto".

\subsection{Measurement Set-Up}

Q-Foto has been developed to automatically verify the correct assembly of a 12-m long parabolic trough steel structure. In the current version, Q-Foto uses a 12-megapixel Nikon D2X-Camera and a motorized positioning system for the camera on a curved track with pan-tilt head and camera rotating unit. It includes the automatic camera control and positioning, photo evaluation and data post-processing. Figure 2 illustrates the principal layout of the system; Figure 3 shows photos of the measurement process.

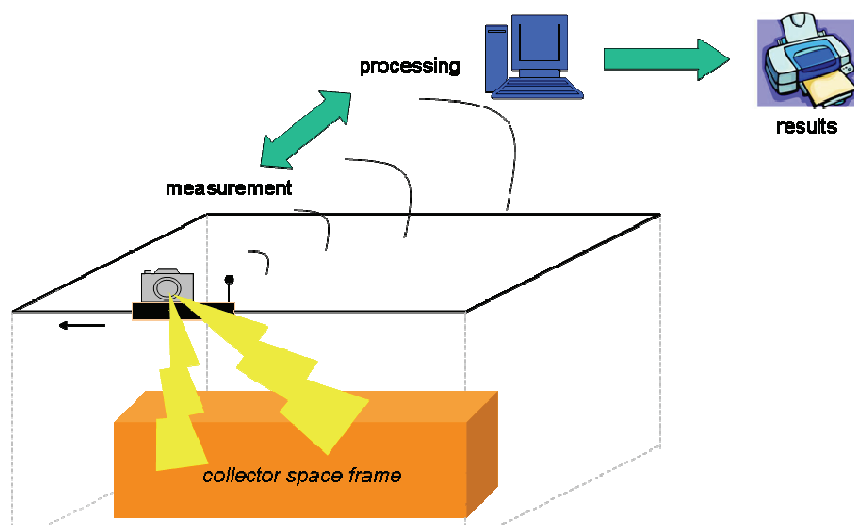

Figure 2: Layout of the Q-Foto system. The camera travels computer controlled along the curved track and takes and transmits measurement images automatically. On completion of one loop the images are evaluated and results are displayed and stored for quality control purposes.
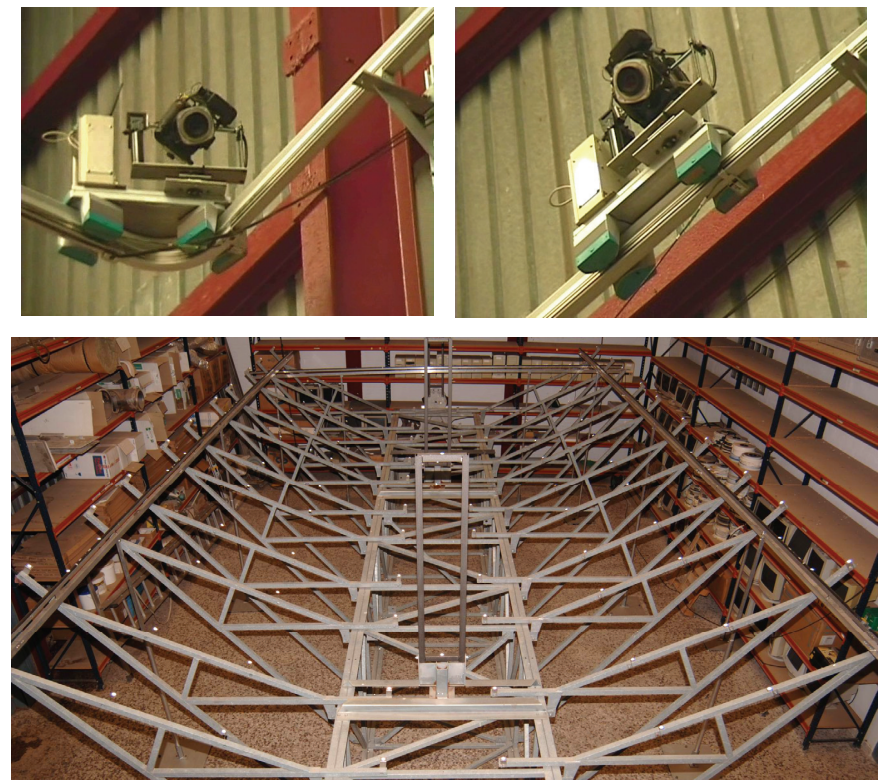

Figure 3: Camera shuttle in different measuring positions (top), and space frame with targets on cantilever arms and receiver supports (bottom)

Using a single high-definition camera and camera selfcalibration, the system allows for high measurement precision, 
even under changing ambient temperature. This is advantageous over systems with stationary cameras that have to be calibrated individually. With the collector space frame placed in the measurement area, retro-reflecting targets are put on the mirror attachment points, receiver supports and reference points and a number of photos are shot. The evaluation results are stored and deviations from the specifications can be identified for adjustment. The required measurement time for analysis, display and storage of the data is about five minutes. Overall cycle time, including target mounting, is below 30 minutes.

\section{$2.2 \quad$ Required measurement precision}

The most relevant assembly errors reducing the intercept factor (percentage of reflected rays that hit the absorber) of the concentrator are deviations of the facets from the parabolic geometry. For the optical efficiency of a collector module, average values for the assembly deviations are considered. An error analysis was presented in (9) to estimate the required measurement accuracies for this application. A relative measurement precision of about $1 / 100.000$ is needed for a $12 \mathrm{~m}$ long module to reach appropriate accuracies. In an on-site production with changing weather conditions this may be difficult to achieve. To check tolerance limits of single points, the measurement uncertainty should be in the order of $1 / 10$ to $1 / 5$ of those tolerance limits. This means that tolerances with a standard deviation of $2 \mathrm{~mm}$ should be measured with a measurement accuracy of about 0.2 to $0.4 \mathrm{~mm}$. A simulation study presented in (9) was done to compute the necessary camera positions to reach these values. The study assisted in the design of the measurement geometry and confirmed that a rectangular camera path surrounding the measurement volume (Figure 2) yields the best combination of measurement accuracy and constancy.

\subsection{System qualification}

The quality and the measurement uncertainties of the Q-Foto measurement system were certified according to the German technical rule VDI/VDE 2634 (10) for optical pointmeasuring devices. The qualification test was performed with measurement targets, whose distances are much more precisely known than the photogrammetric system is able to measure. The targets were fixed on carbon-fiber-reinforced polymer rods with very low temperature expansion coefficient (approximately $-5 \times 10^{-7} / \mathrm{K}$ ) and lengths up to $12 \mathrm{~m}$. With these rods, a network was built up that covered the measurement volume $\left(12 \times 6 \times 1.5 \mathrm{~m}^{3}\right)$. It is displayed in Figure 4. A Laser Doppler displacement meter was used for gauging the distances between the targets on these rods with an uncertainty below $0.05 \mathrm{~mm}$. To account for temperature changes inside the rods, their temperature was measured with thermocouples and corrections for thermal expansions were calculated.

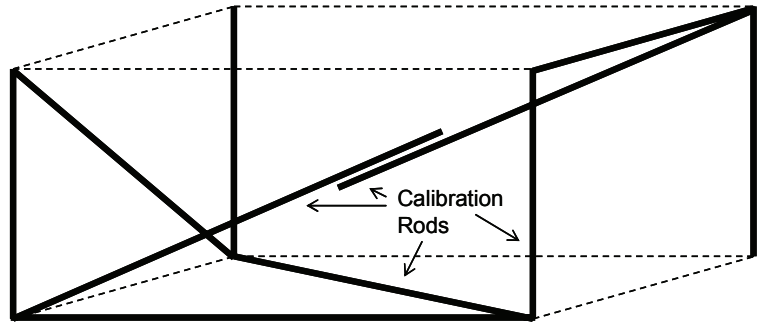

Figure 4: Schematic view of the used certification network of carbon-fiber-reinforced calibration rods according to technical rule VDI/VDE 2634

Differences between the rod lengths measured by the distance meter and the photogrammetry system are used to determine the uncertainty of the photogrammetry system (Figure 5). The largest uncertainty of all single distance measurements is below $\pm 0.4 \mathrm{~mm}$ while the standard deviation reaches $0.1 \mathrm{~mm}$. Based on these experimental results, further simulation studies were performed to investigate possible improvements due to optimized camera viewing angles and paths. According to these simulations, a further reduction of the maximal measurement deviation by $28 \%$ would be possible, so that a maximal uncertainty of well below $0.3 \mathrm{~mm}$ can be reached for the final system.

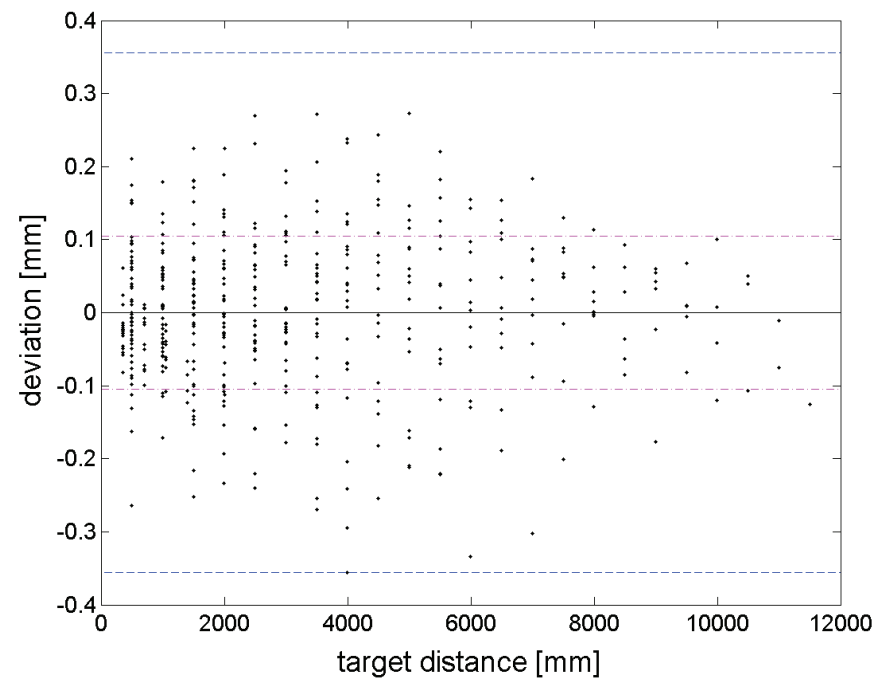

Figure 5: Results of the certification measurements. The graph shows the deviations of the photogrammetric distance measurements from the reference values in $\mathbf{~ m m}$

Due to the qualification of the system according to VDI/VDE 2634, the functionality of the system has been proved. The long-time measurement uncertainty can be tracked with carbon-reinforced rods in the measurement area of the collector module. 


\section{REFLECTOR SHAPE ANALYSIS}

A method for high resolution measurements of surface slope errors of completely assembled trough concentrators was developed. Its basic principle is based on the distant observer method applied since the early 80s (11). The advantage of this method called TARMES (Trough Absorber Reflection Measurement System) over other techniques for similar applications is the quick and easy application. A description of the measurement principle was published before (12). This paper briefly describes the principle to give the necessary background and then focuses on the improvements and extensions made during the continued development.

\subsection{Measurement Set-Up}

\section{Geometry}

Figure 6 shows a sketch of the used measurement set-up. A digital camera is placed at a certain distance of the concentrator on its optical axis and takes images of the mirror facets with the reflection image of the absorber tube (see also Figure 7). The displayed rays in Figure 6 are the ones that come from the upper and lower edges of the reflected image of the absorber tube.

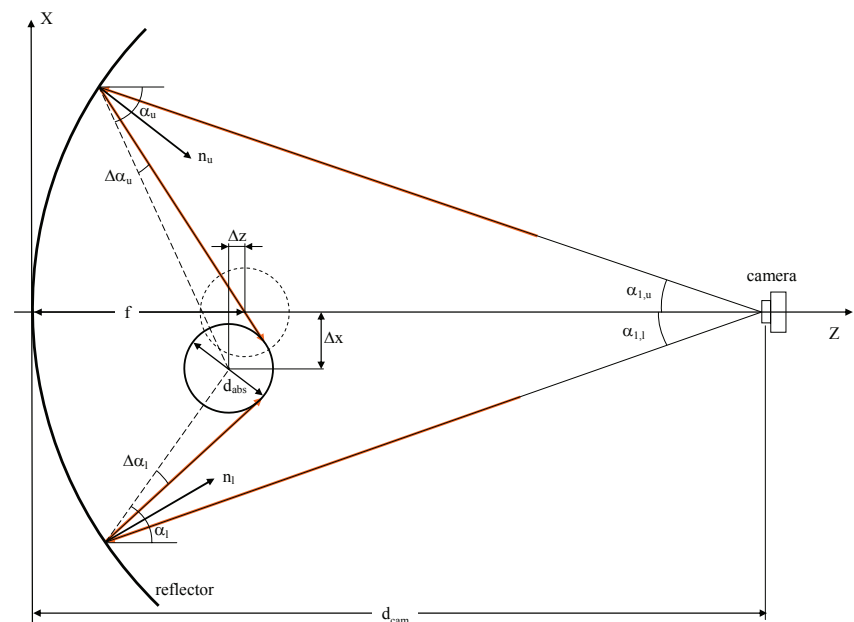

Figure 6: Sketch of the TARMES measurement set-up: the concentrator with absorber tube (left), digital camera (right) and relevant ray paths

When the positions of the camera, the absorber tube and the reflected edges on the concentrator surface are known, the normal vectors of the reflecting surface at the reflected edges can be calculated using geometrical optics. For these calculations the concentrator shape can be assumed to be the ideal parabola. Possible deviations of $\Delta x$ and $\Delta z$ of the absorber tube position from the ideal position due to manufacturing inaccuracies, gravity sag and temperature expansion are measured and considered in the evaluation.

To get information over the whole concentrator surface, the position of the absorber reflection image within the concentrator is varied by rotating the concentrator in defined steps.

\section{Measurements}

The required measurement distance of the camera was reduced from about 100 times to 10 times the focal length. This allows measurements within the collector rows of existing solar fields. The camera is set on a tripod and aligned approximately perpendicular to the trough axis. Its distance to the collector vertex is then measured with a laser distance meter. Before starting the measurement, the collector is tilted up until the reflection of the absorber tube disappears completely. Then a series of pictures is taken while tilting the collector down until the reflection disappears completely on the other side. Figure 7 shows example images of such a series of photos for just three different collector angles.
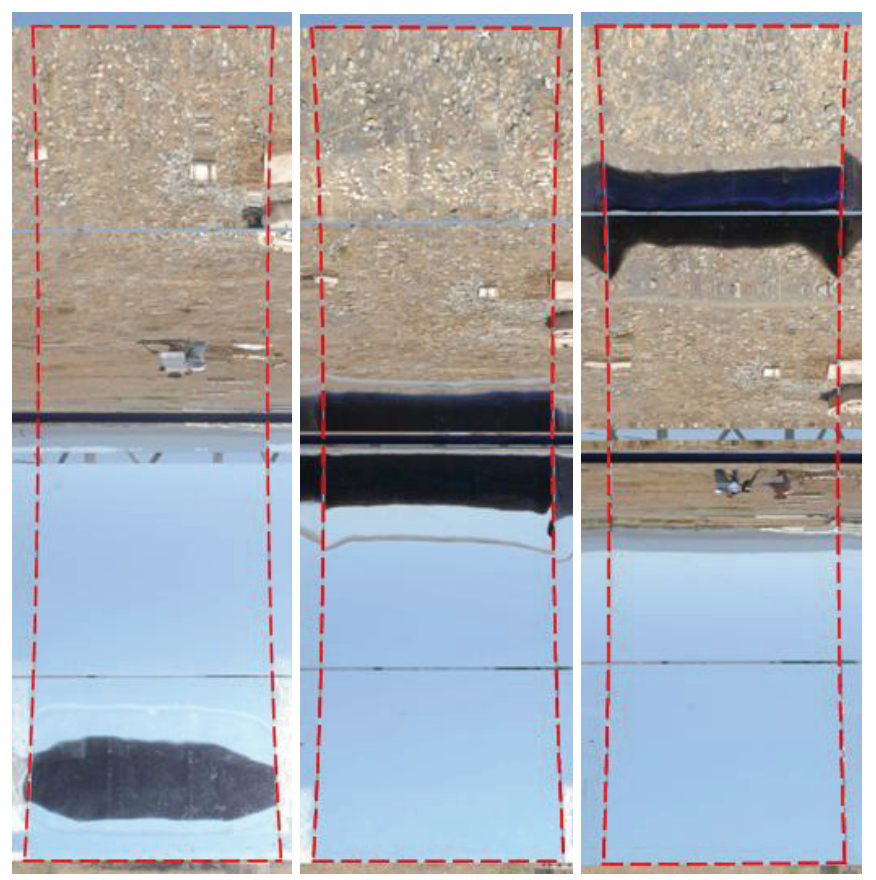

Figure 7: Measurement images of a EuroTrough facet row with the reflection image of the absorber tube in different positions

\subsection{Evaluation}

The images are processed in the image analysis environment Optimas ${ }^{\circledR}$. First, the implemented algorithm corrects the geometrical projection of the three dimensional parabolic shape on the image plane of the camera. These distortions can be seen in the dotted facet outlines in Figure 7 as the facet row has a waisted shape in the images; it appears wider on the side of the collector that is closer to the camera due to the tilt. Then it detects the upper and lower edges of the absorber tube reflection in the images. Areas behind the absorber tube in the center and the gaps between the facets are not taken into account. An automatic control of plausibility 
reduces the influences of erroneously detected edges due to dusty facets, reflected dark objects on the ground or in the sky and other effects to a minimum. The result of the detection process are two matrices of detected lines - one for the upper and one for the lower edge - that are exported with the information of the respective collector angle for further evaluation. Figure 8 visualizes isolines of one of these matrices. The detected edges are shown as colored lines, whereas the facets are displayed in white, gaps in black and manually excluded areas in grey.

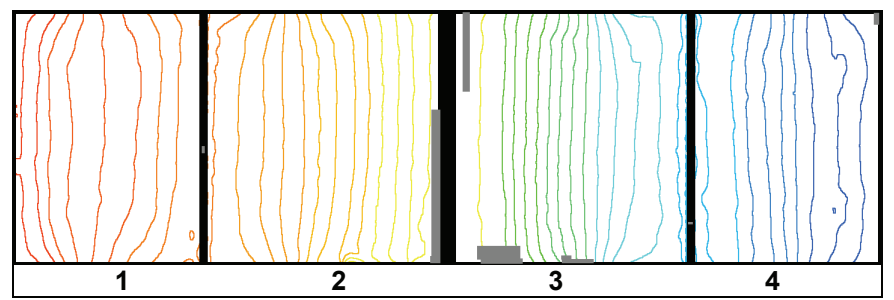

Figure 8: Graph of all detected lines for the upper edge containing the information of the respective collector angle for further evaluation. Compared to the images in Figure 7 the graph was rotated by 90 degrees to the right

The image with zero collector angle relative to the camera is estimated from the series of photos: the one with a symmetrical reflection of the absorber tube in the center is selected. It is not crucial to choose the correct image since the exact tilt angle is later calculated numerically with a parabola fit.

The mathematical computation of the surface normals is implemented in an algorithm that calculates the angles shown in Figure 6 for each data point considering the measured distance and the given parabolic shape and absorber tube diameter. Areas between the detected lines are interpolated. From the two independent data sets of the upper and lower receiver tube edges, a check for inconsistencies is carried out and single outlaying data points can be eliminated.

\section{Error Analysis}

Calculations of the influence of inaccuracies in the measurements of the geometrical distances within the set-up on the measurement result were performed according to the GUM standard (13). Assuming typical geometry and measurement errors for a EuroTrough collector including the remaining uncertainty in absorber position, the measurement uncertainty in the surface slope is $\pm 0.6 \mathrm{mrad}$. This refers to the maximum global error; local dents, waves and other deviations are measured with higher accuracy relative to its surroundings. Uncertainty in the camera distance has only little influence on the total measurement uncertainty.

\subsection{Results}

The measurement method TARMES was applied at one of the EuroTrough prototypes installed at PSA. A mirror area consisting of four facets was measured with TARMES and with high-resolution photogrammetry for reference. Figure 9 shows the slope deviations from the ideal parabola in milliradians measured with TARMES. The sign convention for the slope errors has been defined positive for reflected rays passing above the focal line and negative for reflected rays passing below the focal line. The area covered by the measurements is about $91 \%$ of the total mirror area. The outer areas of the facets show the largest deviations from the ideal slope of up to +8 and $-10 \mathrm{mrad}$. The inner edges of the inner facets are bent down. Both inner facets show a similar local error characteristic, although in facet 3 (counted from the left) it is much more pronounced than in facet 2. The outer facets show smaller deviations with their highest values of about $\pm 4 \mathrm{mrad}$ at the corners and inner edges.

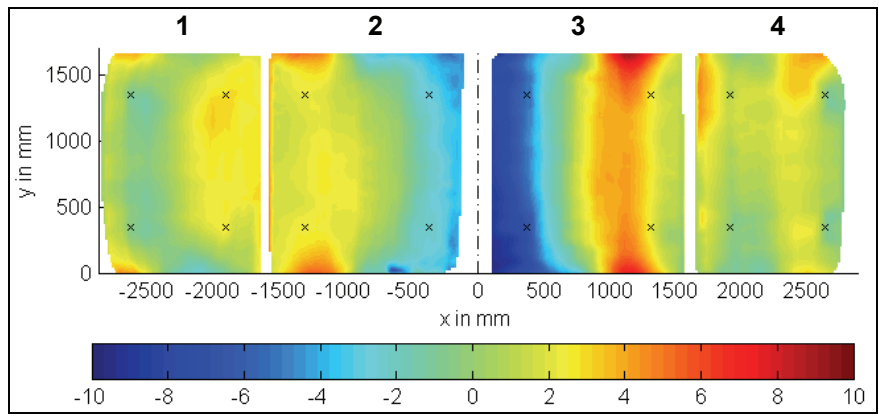

Figure 9: Transversal slope errors (in x-direction) of EuroTrough facets in milliradians measured with TARMES

The histograms of the slope errors of the single facets and the derived statistical errors are given in Figure 10. It can be seen that especially the inner facets do no exhibit a Gaussian error distribution. This makes it difficult to characterize them and simulate their optical behavior with a single Sigma-value. The outer facets are generally better and show closer to Gaussian error distributions. But facet 1 has its peak in the error distribution clearly outside of its center.

The mean value of a single facet gives the deviation of the orientation of this facet, the standard deviation is a measure for the errors of the facet itself and the RMS-value (root mean square) gives the total error of the facets combining both influences. With $4.3 \mathrm{mrad}$, facet 3 exhibits the highest total errors, followed by facet $2(2.3 \mathrm{mrad})$ and the outer facets $(1.7$ mrad). In this particular case the errors are caused mainly by deformation of the facets (standard deviations between 1.1 and $4.3 \mathrm{mrad}$ ) and not from wrong facet orientation (all mean values are below $1.2 \mathrm{mrad}$ ). The RMS-value of the complete facet row is $2.8 \mathrm{mrad}$. It has to be emphasized that the measured deformation values of the facets may have two fundamental causes: shape deviations of the facets themselves and due to assembly errors of the space frame, where the facets are mounted on. Therefore, for the realization of high intercept values it is important to control the quality of the space frame, e.g. with the Q-Foto system. 


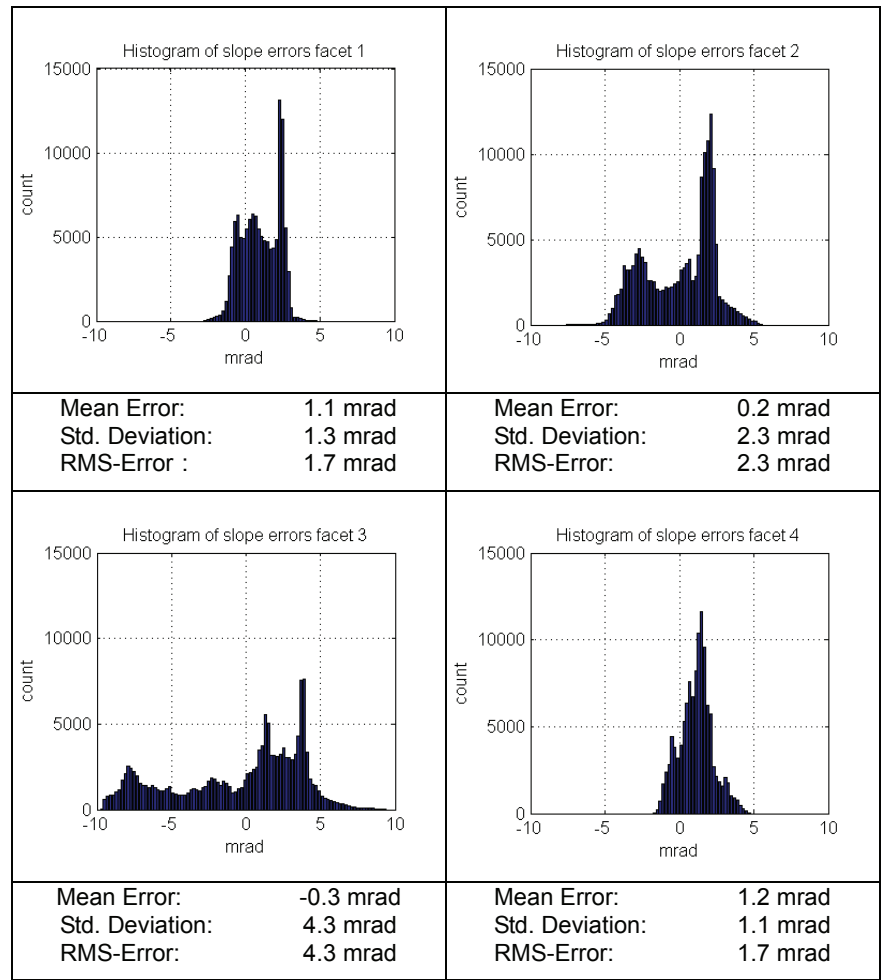

Figure 10: Histograms of slope errors and the derived statistical errors of the measured facets

\section{Comparison to Photogrammetry}

The same four facets were measured by close-range photogrammetry. Results are given in Figure 11.

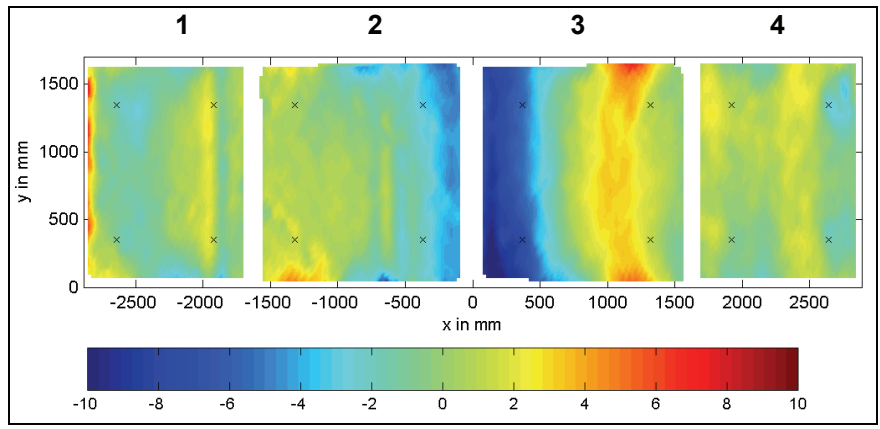

Figure 11: Transversal slope errors (in $x$-direction) of the facets in milliradians, from photogrammetric data

Comparing Figure 9 to Figure 11, good general agreement of the measured slopes can be observed. Minor local differences between the two measurements occur in the upper part of facet 4 and in the left two facets. In the last case shadows during photogrammetry measurements caused inaccuracies. The RMS-value for the complete facet row is 2.7 mrad which agrees well with the TARMES result. The differences of the two measurements in the RMS-values of the single facets are, except for facet 4 , below $0.2 \mathrm{mrad}$. In general, the agreement of the statistical slope errors is very good.

\subsection{Raytracing}

As an additional feature for the complete assessment of parabolic troughs, a raytracing tool was developed that calculates the intercept factor for the given high-resolution measurement data. It calculates the local intercept of every measurement data point considering a defined sunshape. In this case, a universal sunshape with a circum-solar ratio of 0.035 , divided in 19 bins was used. Besides the choice of the sunshape, the raytracing code allows free positioning of the absorber tube and free choice of the receiver diameter, solar incident angle and tracking offset. No glass tube is considered in the code.

Figure 12 shows the resulting local intercept factor for the measured facets (TARMES data) with an absorber tube diameter of $70 \mathrm{~mm}$ and no additional error sources $(0 \mathrm{mrad}$ tracking offset, receiver in design position). It can be seen that, except for very small areas in facet 3 , almost the whole mirror area reflects the incoming sunlight onto the receiver. The total intercept factor is $99.0 \%$, which is a very good value.

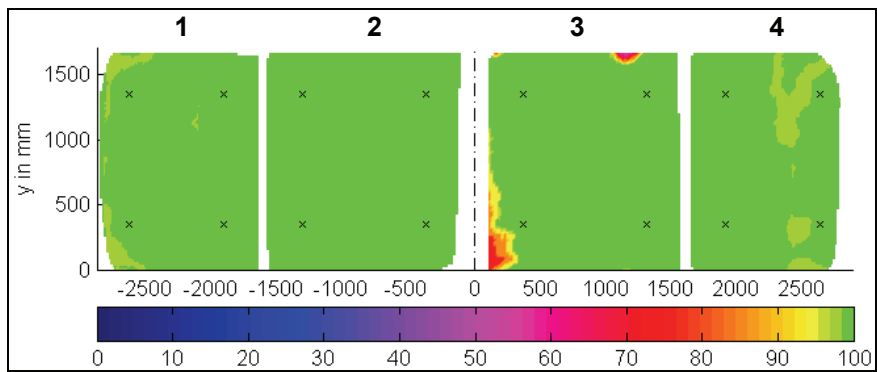

Figure 12: Local intercept factor of the measured facets (TARMES data) for $70 \mathrm{~mm}$ absorber tube diameter, CSR $0.035,0 \mathrm{mrad}$ tracking offset, receiver in design position

The effect of concentrator shape errors on the optical performance becomes more noticeable when the other error influences are also considered. For the present case, the deviation of the absorber tube position in the concentrator from its design position was measured because it is an input for the data evaluation. The deviation in $x$-direction was determined to $+13.5 \mathrm{~mm}$ at $y=0 \mathrm{~mm}$ and $+21.5 \mathrm{~mm}$ at $y=1700 \mathrm{~mm}$. These relatively large deviations are due to inaccuracies in the position of the receiver supports and additionally due to a bent absorber tube. In the ray-tracing model, the $\mathrm{x}$-deviations were supposed to be linear between these points. The deviation in $z$ direction depends on the operation temperature and was supposed to be zero. A value of $1 \mathrm{mrad}$ was chosen as a reasonable error in tracking (accounting for sun algorithm or sun sensor, drive positioning and time steps, structural alignment errors). The raytracing result for the same facets with these parameters is shown in Figure 13.

With these additional error sources it can be seen that the slope errors gain much more significance, as large areas of the facets do not reflect the sunlight onto the receiver. The resulting intercept factor for this case is only $92.7 \%$, which is below the 
expected average value of $96-97 \%$. This example shows that the evaluation of the optical quality of concentrators needs to consider all error influences at the same time. Assessments based on single error types might overestimate optical performance.

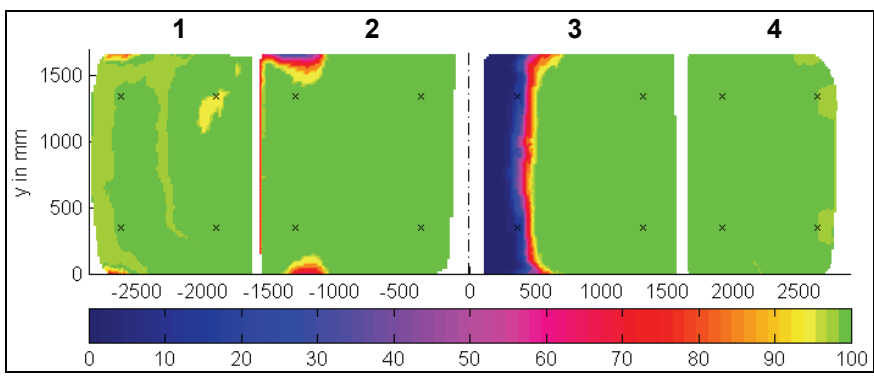

Figure 13: Local intercept factor of the measured facets (TARMES data) for $70 \mathrm{~mm}$ absorber tube diameter, CSR $0.035,1$ mrad tracking offset, receiver in deviated position

\section{CONCLUSIONS}

The work has shown, that starting off from mobile photogrammetric applications on the EuroTrough prototype collector to analyze features of its reflector geometry, the measurement technique has been refined to a fast, automatic, robust and calibrated tool for collector assembly quality control. Accuracy of the described Q-Foto system reaches levels of tenths of a millimeter for assuring high optical performance levels of the collectors.

A technique derived from the distant observer method has been developed and adapted for reflector slope measurements on parabolic trough collectors in a solar field. It now requires distances of less than 10 times the focal length and uses image analysis tools to determine the reflector curvature and its alignment in the concentrator. Although it should not replace proper adjustment and quality control during the assembly, it can be used to check the achieved optical quality and to identify and exactly locate geometric deficiencies of a solar concentrator within short time. Beyond the reflector slope errors, the ray-tracing results have helped to quantify the impact of geometry tolerances on the optical efficiency and to deduce the necessary specifications for reaching high intercept factor values. Its application range is for prototype evaluation as well as series production quality control.

\section{ACKNOWLEDGMENTS}

Financial support from the German Federal Ministry for the Environment, Nature Conservation and Nuclear Safety (OPAL-d1, 16UM0045) is gratefully acknowledged.

\section{REFERENCES}

(1) Lüpfert E, Pottler K, Schiel W, Optimization of cost and efficiency in concentrating solar power technology trough quality control in large production series for solar fields. In:
Proceedings of the ISES, EuroSun Congress; June 20-23; Freiburg, Germany. 2004.

(2) Shortis MR, Johnston G, Photogrammetry: An available surface characterization tool for solar concentrators, Part I: Measurement of surfaces. Journal of Solar Energy Engineering. 1996; 118: 146-150.

(3) Shortis MR, Johnston G, Photogrammetry: An available surface characterization tool for solar concentrators, Part II: Assessment of surfaces. Journal of Solar Energy Engineering. 1997; 119: 286-291.

(4) Pottler K, Lüpfert E, Johnston G et al, Photogrammetry: A powerful tool for geometric analysis of solar concentrators and their components. Journal of Solar Energy Engineering. 2005; 127: 94-101.

(5) Lüpfert E, Pottler K, Ulmer S et al, Parabolic trough performance analysis techniques. In: Proceedings of Internat. Solar Energy Conf. (ISEC 05), Orlando, FL, USA, 2005.

(6) Wendelin T, May K, Gee R, Video Scanning Hartmann Optical Testing of State-of-the-Art Parabolic Trough Concentrators. In: Proceedings of Solar 2006 Conference (ISEC '06), July 8-13, 2006, Denver, Colorado, USA, 2006.

(7) Maccari A, Montecci M, An optical profilometer for the characterization of parabolic trough solar concentrators. In: Solar Energy 81, pp. 185-194, 2006.

(8) Kammel S, Deflectometry for quality control of specular surfaces. Technisches Messen tm 2003(4);70:193-8.

(9) Pottler K, Röger M, Lüpfert E, Schiel W, Automatic noncontact quality inspection system for industrial parabolic trough assembly. In: Proceedings, 13th International Symposium, Concentrated Solar Power and Chemical Energy Technologies, June 20-23, 2006, Seville, Spain, ISBN: 84-7834-519-1.

(10) VDI/VDE 2634, Part 1, Optical 3D measuring systems, Imaging systems with point-by-point probing. ICS 17.040.30, 2002.

(11) Wood RL, Distant Observer Techniques for Verification of Solar Concentrator Optical Geometry. UCRL-53220, Lawrence Livermore Laboratory, University of California, 1981.

(12) Ulmer S, Heinz B, Pottler K, Lüpfert E, Slope Error Measurements for Parabolic Troughs Using the Reflection Image of the Absorber Tube. In: Proceedings of the 13th SolarPACES International Symposium on Concentrated Solar Power and Chemical Energy Technologies, June 20-23, 2006, Seville, Spain, ISBN: 84-7834-519-1.

(13) DIN V/ENV 13005: Guide to the expression of uncertainty in measurement. Vornorm DIN V/ ENV 13005, Beuth Verlag, Berlin, 1999. 